\title{
Partonic effects on the elliptic flow at relativistic heavy ion collisions
}

\author{
Zi-wei Lin and C. M. Ko \\ Cyclotron Institute and Physics Department, Texas A\&M University, College Station, Texas 77843-3366
}

(Received 16 August 2001; published 14 February 2002)

\begin{abstract}
The elliptic flow in heavy ion collisions at the Relativistic Heavy Ion Collider is studied in a multiphase transport model. By converting the strings in the high energy density regions into partons, we find that the final elliptic flow is sensitive to the parton scattering cross section. To reproduce the large elliptic flow observed in $\mathrm{Au}+\mathrm{Au}$ collisions at $\sqrt{s}=130 \mathrm{~A} \mathrm{GeV}$ requires a parton scattering cross section of about $6 \mathrm{mb}$. We also study the dependence of the elliptic flow on the particle multiplicity, transverse momentum, and particle mass.
\end{abstract}

DOI: 10.1103/PhysRevC.65.034904

PACS number(s): 25.75.Ld, 24.10.Lx

\section{INTRODUCTION}

Elliptic flow in heavy ion collisions measures the asymmetry of particle momentum distributions in the plane perpendicular to the beam direction. It results from the initial spatial asymmetry in noncentral collisions [1,2]. Theoretical studies have shown that the elliptic flow is sensitive to the properties of the hot dense matter formed during the initial stage of heavy ion collisions [3-6].

Recently, elliptic flow has been measured at the Relativistic Heavy Ion Collider (RHIC) in $\mathrm{Au}+\mathrm{Au}$ collisions at $\sqrt{s}=130 A \mathrm{GeV}$. A large elliptic flow of all charged particles near midrapidity was reported by the STAR Collaboration [7]. Also, the transverse momentum dependence of the elliptic flow of all charged particles was measured by both the STAR [7] and the PHENIX [8] Collaborations, while those of charged pions, kaons, protons, and antiprotons were measured by the STAR Collaboration $[9,10]$. The experimental results show that the elliptic flow first increases with particle transverse momentum and then levels off. The dependences of elliptic flow on both the charged particle multiplicity $[7-9,11]$ and the particle pseudorapidity [11] have also been measured.

To understand these experimental results, many theoretical approaches have been used. These include semianalytic models [12], models with parton energy loss [13,14], hydrodynamic models $[3,15,16]$, transport models $[4,17]$, and the hybrid model which combines hydrodynamic and hadronic transport models [18]. Among these studies, hydrodynamic models usually give the largest elliptic flow and an almost linear increase in its value with the particle transverse momentum below $1.5 \mathrm{GeV} / c$. In the hybrid model of combining the hydrodynamic model with the RQMD transport model [19] and choosing certain effective equation of state, it is possible to obtain an elliptic flow that is comparable to the measured ones in heavy ion collisions at both SPS and RHIC energies [18]. In transport models including only the parton cascade, the elliptic flow has been shown to be sensitive to the parton scattering cross section, and a large value can be obtained with a large cross section $[20,21]$. On the other hand, transport models based on hadronic and/or string degrees of freedom in general give a smaller elliptic flow [9] than that observed at RHIC. Including multi-Pomeron exchanges and hard gluon-gluon scatterings can, however, yield a large elliptic flow [17].
In this paper, we study the elliptic flow in $\mathrm{Au}+\mathrm{Au}$ collisions at RHIC using a multiphase transport model (AMPT) that includes both initial partonic and final hadronic interactions as well as the transition between these two phases of matter [22-24]. In particular, we study the effect due to partons converted from the initial strings in high energy density regions.

\section{CONVERSION OF STRINGS TO PARTONS}

In most transport models for heavy ion collisions, such as the ART model [25], the RQMD model [19], and the UrQMD model [26], initial primary collisions produce either hadrons or strings which later fragment to hadrons. In the HIJING model [27], on the other hand, initial primary collisions also produce minijet partons which later enter into the string configurations and fragment to hadrons. These minijet partons are partons produced from initial hard collisions, i.e., from perturbative QCD processes involving a minimum transverse momentum transfer $p_{0}$, which is chosen to be $2 \mathrm{GeV} / c$ in the HIJING model to reproduce the $p p$ and $p \bar{p}$ data [27].

In the AMPT model [22-24], which takes initial conditions from the HIJING model, minijet partons first undergo scatterings before fragmenting into hadrons. Since the number of hard collisions in an $A+A$ collision roughly scales as $A^{4 / 3}$ and grows faster with colliding energy, while the number of strings roughly scales as $A$, minijets become more important in heavy ion collisions at higher energies. However, for central $\mathrm{Au}+\mathrm{Au}$ collisions even at $200 \mathrm{~A} \mathrm{GeV}$ minijet partons account for only about $1 / 3$ of the total produced transverse energy, so the effect of parton scattering on the final particle multiplicities and spectra is quite small $[23,24]$.

The above picture of coexisting partons and strings during the initial stage of high energy heavy ion collisions is questionable when the energy density is much higher than the critical density for the QCD phase transition. In this case, the strings are expected to melt into partonic degrees of freedom. Both the transport model [28] and the high density QCD approach [29] predict that the initial energy density of produced matter in central $\mathrm{Au}+\mathrm{Au}$ collisions at RHIC is more than an order of magnitude higher than the critical energy density $\left(\sim 1 \mathrm{GeV} / \mathrm{fm}^{3}\right)$. Keeping strings in the high energy density region thus underestimates the partonic effects in these collisions. 
To model the string melting in high energy density regions to partons, we extend the AMPT model in the following way. After using the HIJING model (with jet quenching turned off) to produce the initial conditions, we first let strings fragment to hadrons using the LUND fragmentation $[30,23]$ built in the PYTHIA routine [31] and then convert these hadrons to partons according to their flavor and spin structure. In particular, a meson is converted to a quark and an antiquark, while a baryon is converted to three quarks, and an antibaryon is converted to three antiquarks, where quark masses are taken as the same as in the PYTHIA program [31], e.g., $m_{u}=5.6 \mathrm{MeV}, m_{d}=9.9 \mathrm{MeV}, m_{s}=199 \mathrm{MeV}$. We further assume that quarks are produced isotropically in the rest frame of the hadron and start to interact only after a proper formation time $\tau_{0}^{P}$. In converting hadrons to partons, hadrons are not assigned a formation time as they are considered as an intermediate step in modeling the melting of strings to partons in an environment of high energy density. Based on the expectation that hard partons (e.g. those described by perturbative QCD) are produced early while soft partons are produced late in the process, we take $\tau_{0}^{P}=1 / Q$ for the parton proper formation time, where $Q$ is a scale related to the parton transverse momenta. Since partons produced from a string through the same intermediate hadron should have the same formation time, the transverse mass of the hadron is thus used for $Q$ in determining the parton formation time.

The scatterings among these quarks are treated using the parton cascade ZPC with the following universal cross section [32]:

$$
\frac{d \sigma_{p}}{d t}=\frac{9 \pi \alpha_{s}^{2}}{2}\left(1+\frac{\mu^{2}}{s}\right) \frac{1}{\left(t-\mu^{2}\right)^{2}} .
$$

In the above, the strong coupling constant $\alpha_{s}$ is taken to be 0.47, $s$ and $t$ represent the standard Mandelstam variables for the two-parton elastic scattering process, and the effective screening mass $\mu$ depends on the temperature and density of the partonic matter. In the present study, we shall take $\mu$ as a parameter to obtain the desired total cross section and the corresponding angular distribution.

A parton can hadronize after it stops interacting, i.e., after it will no longer collide with other partons. We model the hadronization by combining the nearest two partons into a meson and three partons into a baryon (or an antibaryon). As partons freeze-out at different times and parton coalescence occurs at different times, the hadronization is treated locally. Since combinations of partons form a continuous invariantmass spectrum but not a discreet one, it is in general impossible to conserve four-momentum when several partons are combined into a hadron. In our current treatment, we choose to conserve the three-momentum and determine the hadron species according to the flavor and invariant mass of coalescing partons. For example, if a $\bar{u}$ and a $d$ quark coalesce, a $\pi^{-}$ will be formed if the two-quark invariant mass is closer to the $\pi^{-}$mass, or a $\rho^{-}$will be formed if the two-quark invariant mass is closer to the central value of the $\rho$ mass [33]. All SU(3) mesons and baryons listed in the HIJING program [27] are included for coalescence except $\eta^{\prime}, \Sigma^{*}$, and $\Xi^{*}$, which are not present in our hadronic transport model, and $K_{S}^{0}, K_{L}^{0}$ states. The resulting hadrons are then given an additional formation time of $\tau_{0}^{H}=0.7 \mathrm{fm} / c$ in their rest frame and imported to the ART hadronic transport model to take into account their rescatterings [22-25].

\section{RESULTS ON ELLIPTIC FLOW}

\section{A. Time evolution}

We have used the above extended AMPT model to study the elliptic flow in $\mathrm{Au}+\mathrm{Au}$ collisions at RHIC energies. The elliptic flow here is defined as

$$
v_{2}=\left\langle\frac{p_{x}^{2}-p_{y}^{2}}{p_{x}^{2}+p_{y}^{2}}\right\rangle
$$

where the average is performed over all particles. For centerof-mass energy $\sqrt{s}=130 \mathrm{~A} \mathrm{GeV}$ and impact parameter $b$ $=8 \mathrm{fm}$, we show in Figs. 1(a) and 1(b) the time evolution of the elliptic flow of all partons, i.e., regardless of their formation and freeze-out times, at midrapidity with parton scattering cross sections $\sigma_{p}=3$ and $6 \mathrm{mb}$, respectively. We note that partons still inside strings and partons which have frozen out are also included in Fig. 1. For both values of parton cross sections the elliptic flow is seen to develop mostly within the first $5 \mathrm{fm} / c$, and both the rate of increase and the final parton $v_{2}$ are larger for the larger parton cross section. Also shown in these figures are the time evolutions of the parton transverse energy $E_{T}$ (scaled down by a factor of 2000 ), the average transverse energy per parton $\left\langle E_{T}\right\rangle$ (scaled down by 2), and the second moment of the spatial asymmetry determined from the parton positions at their previous interaction points, i.e., $s_{2}=\left\langle\left(x^{2}-y^{2}\right) /\left(x^{2}+y^{2}\right)\right\rangle$. It is seen that for both parton cross sections the absolute value of $s_{2}$ decreases with time, and its final value is closer to zero in the case of $\sigma_{p}=6 \mathrm{mb}$. The final saturation of parton $v_{2}$ in the partonic phase is due to the lack of scatterings in the limit of small $\sigma_{p}$ and the vanishing spatial anisotropy in the limit of large $\sigma_{p}$. We also see that the transverse energy at midrapidity decreases by $\sim 30 \%$ within a couple of $\mathrm{fm} / c$. Since the decrease of parton transverse energy is faster than that for the parton number at midrapidity, the average transverse energy per parton $\left\langle E_{T}\right\rangle$ also decreases. The final saturation of $\left\langle E_{T}\right\rangle$ at $\sim 20 \%$ below the initial value reflects the equilibration between the longitudinal and transverse momenta of partons [34,35].

In Fig. 2, we show the time evolution of $v_{2}$ for active partons, i.e., partons that have not stopped scatterings, $v_{2}$ for formed hadrons, the total $v_{2}$ and $s_{2}$ including both active partons and formed hadrons, and the number of active partons at midrapidity for collisions at an impact parameter $b$ $=8 \mathrm{fm}$ and a parton cross section $\sigma_{p}=6 \mathrm{mb}$. While the initial numbers of active partons are near zero because most partons are not yet formed and thus not yet active, it is seen that the number of active partons peaks within the first $\mathrm{fm} / \mathrm{c}$ and decreases by a factor of 2 at about $4 \mathrm{fm} / c$ due to the 


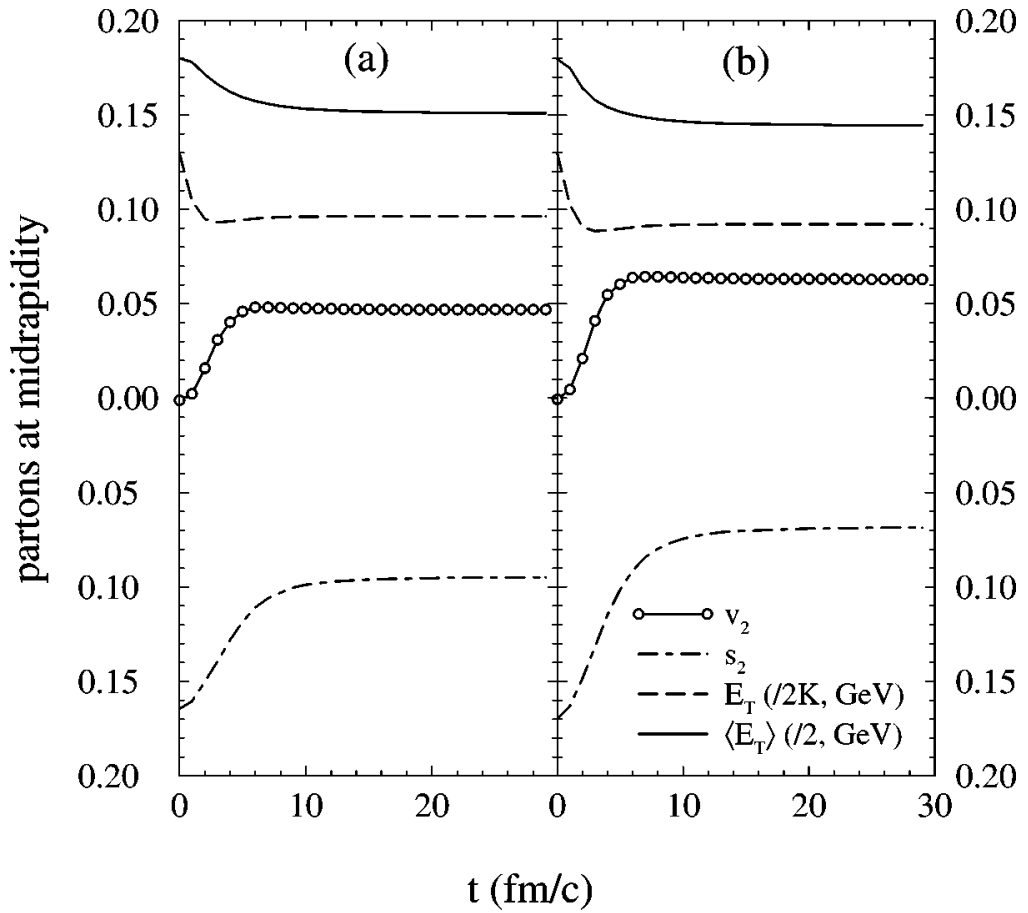

FIG. 1. Time evolutions of the parton elliptic flow, spatial anisotropy, transverse energy, and average transverse energy per parton at midrapidity in $\mathrm{Au}+\mathrm{Au}$ collisions at center-of-mass energy $130 A \mathrm{GeV}$ and impact parameter $b=8 \mathrm{fm}$ for parton scattering cross sections $\sigma_{p}=3 \mathrm{mb}$ (a) and $6 \mathrm{mb}(\mathrm{b})$
, hadronization. The total $v_{2}$ is thus dominated by active partons at the early stage and by formed hadrons during the later stage of heavy ion collisions. Although the active partons have a large elliptic flow at later times, their number is too small to affect the total elliptic flow.

\section{B. Impact parameter dependence}

We have also studied the impact parameter dependence of elliptic flow in $\mathrm{Au}+\mathrm{Au}$ collisions at center-of-mass energies

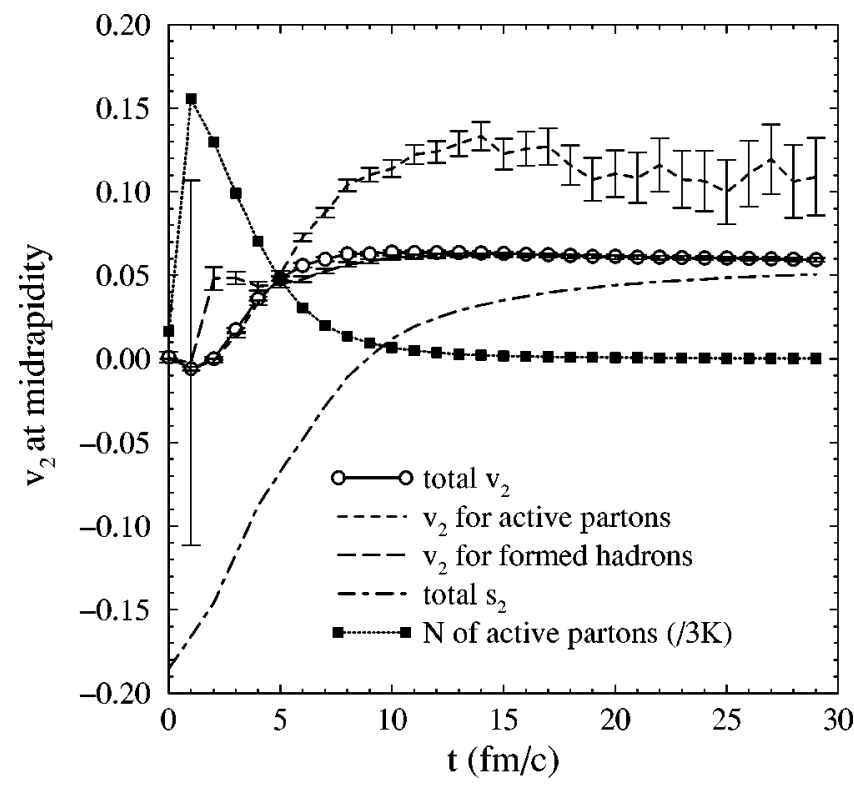

FIG. 2. Time evolutions of elliptic flow, spatial anisotropy, and the number of active partons at midrapidity in $\mathrm{Au}+\mathrm{Au}$ collisions at center-of-mass energy of $130 \mathrm{~A} \mathrm{GeV}$ and impact parameter $b=8 \mathrm{fm}$ for a parton cross section $\sigma_{p}=6 \mathrm{mb}$. of $130 A \mathrm{GeV}$. To compare with the centrality dependence in the STAR data [7], we first divide the impact parameter range $0 \leqslant b \leqslant 13$ fm into six bins with equal bin size except the first bin, which is taken to be $0 \leqslant b \leqslant 3 \mathrm{fm}$. The impact parameter dependence is then converted to the dependence on $N_{\text {ch }} / N_{\text {max }}$ by taking $N_{\text {ch }}$ as the number of charged particles within the pseudorapidity range $\eta \in(-0.75,0.75)$ and $N_{\max }$ as its value at $b=0 \mathrm{fm}$. Furthermore, we include only charged particles within $\eta \in(-1.3,1.3)$ and transverse momentum range $p_{t} \in(0.1,2.1) \mathrm{GeV} / c$ in evaluating the elliptic flow $v_{2}$ in order to compare more directly with the STAR data on the centrality dependence.

In Fig. 3, we show the results for the elliptic flow of charged particles as a function of $N_{\mathrm{ch}} / N_{\max }$ for the scenarios of the default AMPT (without string melting) and the extended AMPT (with string melting). The error bars in our results represent only the statistical error in $v_{2}$ but not that in $N_{\text {ch }}$. Although all results show the qualitative features of the observed centrality dependence of $v_{2}$ [7], the shape and magnitude of $v_{2}$ depend sensitively on the partonic dynamics [36]. Without converting the initial strings into partons, the default AMPT model gives the smallest elliptic flow. Allowing the melting of strings to partons, the elliptic flow for a larger parton cross section not only is higher than that for a smaller partonic cross section, but also peaks at a lower value of $N_{\mathrm{ch}} / N_{\max }$. Of the three parton cross sections, the results for $\sigma_{p}=6 \mathrm{mb}$ appear to be more consistent with the observed centrality dependence of the elliptic flow.

We note that the results from the default AMPT model are insensitive to the parton scattering cross section, which is taken to be $3 \mathrm{mb}$ in the scenario with strings. This is mainly due to the small fraction of energy that is carried by minijet partons and the lack of transverse collective motion of the strings. As a result, the elliptic flow is significantly reduced 


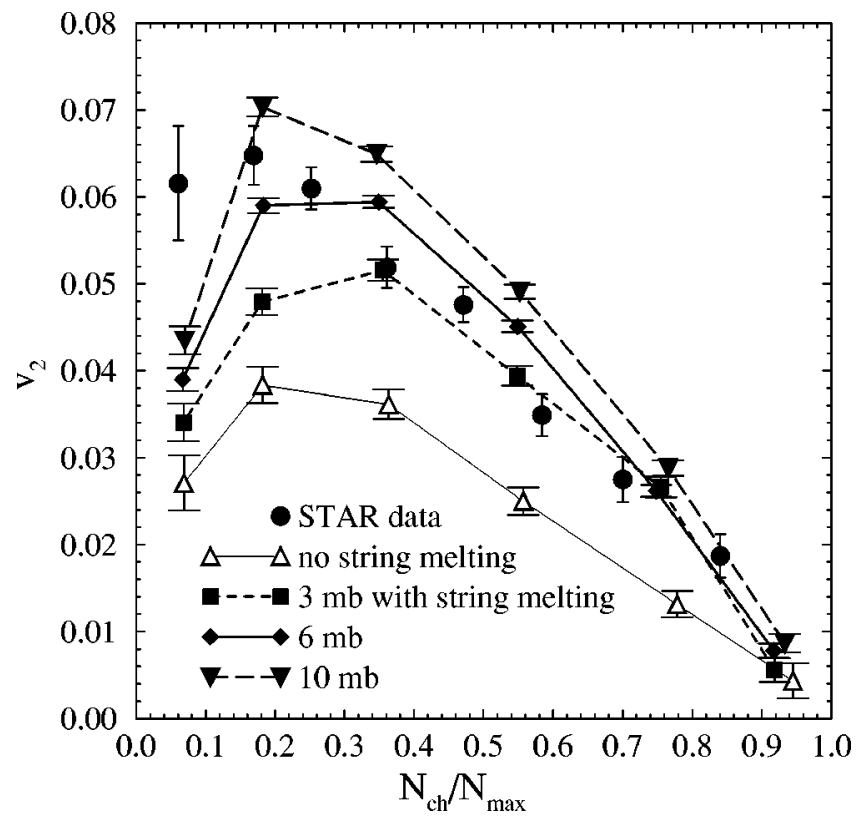

FIG. 3. Impact parameter dependence of elliptic flow at $130 \mathrm{~A} \mathrm{GeV}$. The data from the STAR collaboration [7] are shown by filled circles, while the theoretical results for different partonic dynamics are given by curves.

after minijet partons combine with strings and fragment to hadrons. However, with strings converting to partons, the initial energy originally stored in the strings also contributes to the parton dynamics. This leads to a larger elliptic flow and its sensitivity to the parton cross section, and thus makes it possible to determine the strength of partonic interactions from the final elliptic flow.

\section{Transverse momentum dependence}

Our results for the dependence of charged particle elliptic flow on the transverse momentum, i.e., the differential elliptic flow $v_{2}\left(p_{t}\right)$, are shown in Fig. 4. As observed in the experiment data, the differential elliptic flow first increases almost linearly with transverse momentum and then tends to level off at large transverse momenta. However, both the slope of initial increase and the transverse momentum at which deviation from a linear dependence appears are affected by the parton dynamics. The result from the default AMPT model [22-24] (open triangles), which includes only minijets in the partonic phase, has the smallest $v_{2}\left(p_{t}\right)$ at a given $p_{t}$ and shows a departure from the linear dependence also at the smallest transverse momentum. Including partons from string melting in the high energy density regions increases both the magnitude of $v_{2}\left(p_{t}\right)$ and the value of $p_{t}$ at which the linear dependence breaks down.

The differential elliptic flow $v_{2}\left(p_{t}\right)$ is expected to be different for different particles [37,15]. Figure 5 shows our results for pions, kaons, and nucleons from the extended AMPT model together with the STAR data for charged particles [7]. At low transverse momentum $\left(p_{t}<1 \mathrm{GeV} / c\right)$, while the $v_{2}\left(p_{t}\right)$ of pions and kaons increases almost linearly with $p_{t}$, that of nucleons shows a stronger dependence on $p_{t}$. Furthermore, particles with smaller masses are seen to

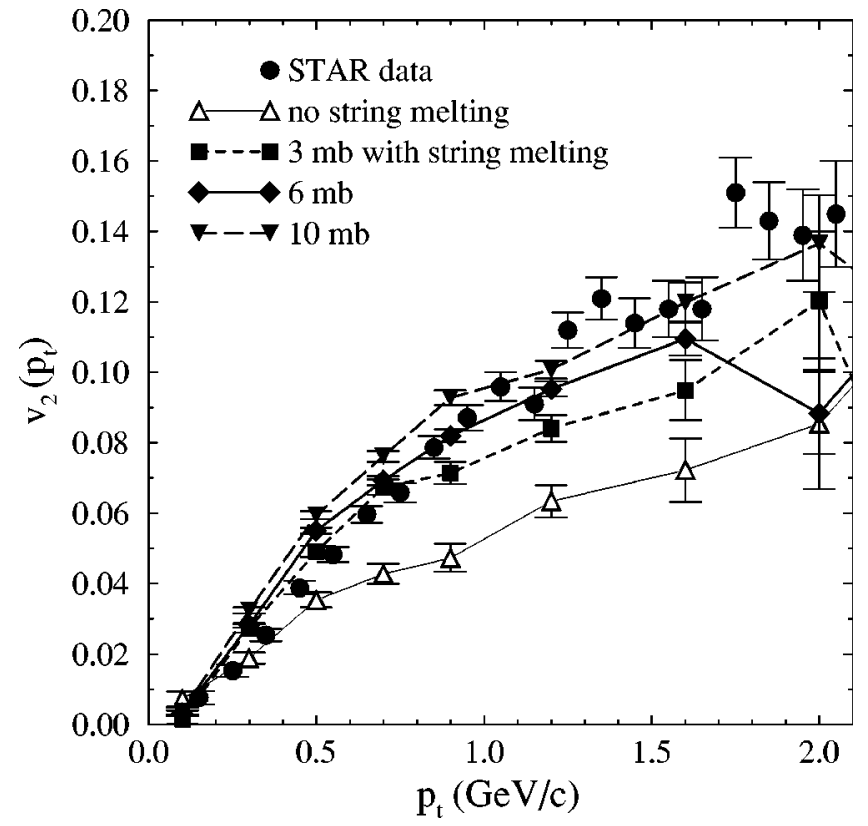

FIG. 4. Transverse momentum dependence of elliptic flow at $130 A \mathrm{GeV}$. Circles are the STAR data for minimum-bias $\mathrm{Au}+\mathrm{Au}$ collisions [7], and curves represent the minimum-bias results for charged particles within $\eta \in(-1.3,1.3)$ from the AMPT model.

have higher values of $v_{2}\left(p_{t}\right)$ at a given $p_{t}$. These features are qualitatively similar to those observed from the STAR data [10] and also those obtained from the hydrodynamic models $[15,16]$. On the other hand, all particles seem to have similar values of elliptic flow, within the errors of our calculations, at a given $p_{t}$ above $1 \mathrm{GeV} / c$.

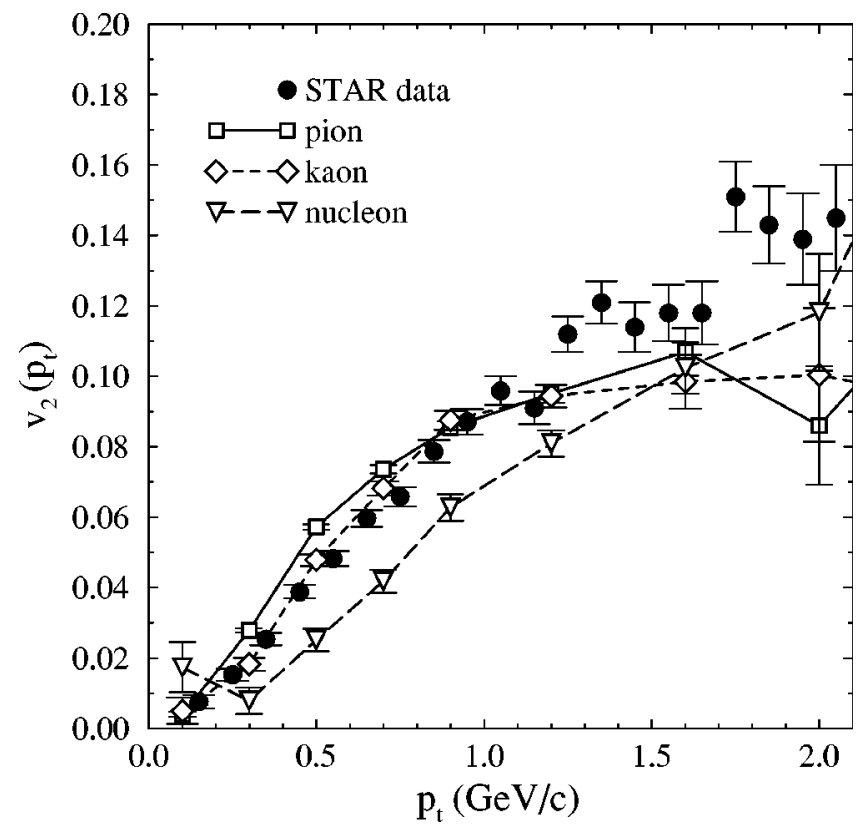

FIG. 5. Transverse momentum dependence of the elliptic flow for particles of different masses in the case of parton cross section $\sigma_{p}=6 \mathrm{mb}$ in $\mathrm{Au}+\mathrm{Au}$ collisions at $130 \mathrm{~A} \mathrm{GeV}$. Curves represent the minimum-bias results within $\eta \in(-1.3,1.3)$ from the extended AMPT model. 


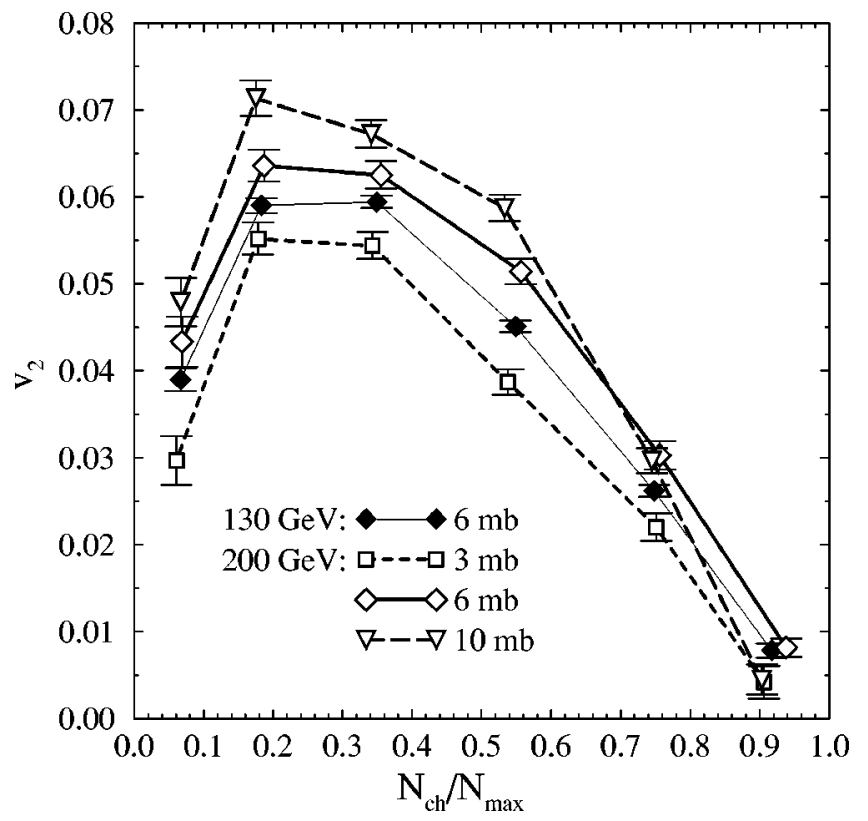

FIG. 6. Impact parameter dependence of elliptic flow at $200 \mathrm{~A} \mathrm{GeV}$. Curves represent minimum-bias results for charged particles within $\eta \in(-1.3,1.3)$ and $p_{t} \in(0.1,2.1) \mathrm{GeV} / c$.

To test the sensitivity of our results to different formation time parameters, we have calculated the elliptic flow at $b$ $=8 \mathrm{fm}$ for the following three cases for comparison: (i) parton formation time $\tau_{0}^{P}$ larger by a factor of 2, (ii) $\tau_{0}^{P}$ smaller by a factor of 2, (iii) hadron formation time $\tau_{0}^{H}$ set to $0.01 \mathrm{fm} / c$ instead of the default $0.7 \mathrm{fm} / c$. Compared to our default results, the relative changes of final charged particle $v_{2}$ are found to be less than $10 \%$ for $p_{\perp}<2 \mathrm{GeV} / c$.

\section{Elliptic flow at $\sqrt{s}=200 A \mathbf{~ G e V}$}

We have also studied the elliptic flow in $\mathrm{Au}+\mathrm{Au}$ collisions at the maximum RHIC energy of $200 A \mathrm{GeV}$ using the extended AMPT model. In Figs. 6 and 7, we show the dependence of the charged particle elliptic flow on impact parameter and transverse momentum, respectively, together with the results for $\sigma_{p}=6 \mathrm{mb}$ at $\sqrt{s}=130 \mathrm{~A} \mathrm{GeV}$ (curves with filled diamonds). Compared to Fig. 3 , it is seen that for the same parton cross section the elliptic flow in less central events $\left(N_{\mathrm{ch}} / N_{\max }<0.6\right)$ increases only slightly (no more than 0.01 in magnitude) with the center-of-mass energy. As a result, the sensitivity of the centrality dependence of elliptic flow to the parton scattering cross section in heavy ion collisions at $\sqrt{s}=200 \mathrm{~A} \mathrm{GeV}$ is similar to that at $\sqrt{s}$ $=130 \mathrm{~A} \mathrm{GeV}$.

For the differential elliptic flow, Fig. 7 shows that for transverse momenta below about $1 \mathrm{GeV} / c$, it shows even less change with the center-of-mass energy than the impact parameter dependence of the elliptic flow. As to the dependence on the parton cross section, the differential elliptic flow seems to show a larger sensitivity at higher transverse momenta. However, to study this quantitatively requires much better statistics than we have obtained so far. Also, for elliptic flow at high $p_{t}$, the effect of energy loss due to

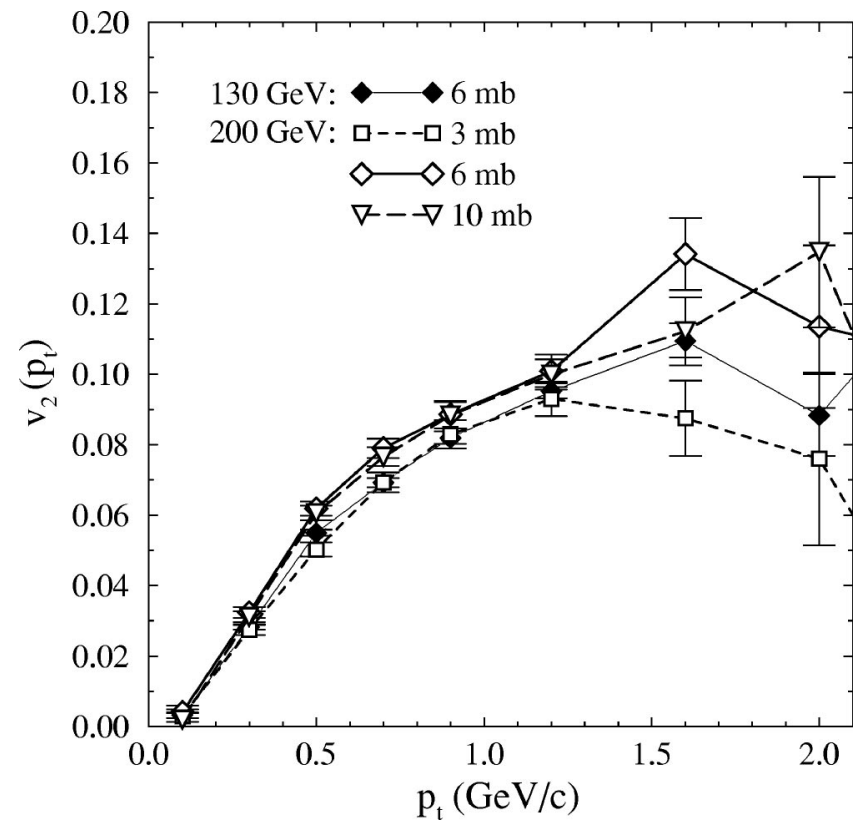

FIG. 7. Transverse momentum $p_{t}$ dependence of elliptic flow at $200 \mathrm{~A} \mathrm{GeV}$. Curves represent minimum-bias results for charged particles within $\eta \in(-1.3,1.3)$.

inelastic partonic processes, which have not been included in the AMPT model, becomes important $[13,14]$. Furthermore, the parton coalescence model may be less suitable than the independent parton fragmentation model for modeling the hadronization dynamics.

\section{CONCLUSIONS AND DISCUSSIONS}

In this paper, we have studied the elliptic flow in heavy ion collisions at RHIC using a multiphase transport model that includes both partonic and hadronic scatterings. To take into account the effects of string melting due to initial high energy density, we have introduced a schematic model to convert the strings produced in soft interactions into partons. We find that the magnitude of the elliptic flow is sensitive to the scattering cross sections of these partons. To reproduce the large elliptic flow observed in $\mathrm{Au}+\mathrm{Au}$ collisions at $130 \mathrm{~A} \mathrm{GeV}$ at RHIC requires a parton scattering cross section of roughly $6 \mathrm{mb}$. Similar to the findings from hydrodynamic models, the differential elliptic flow $v_{2}\left(p_{t}\right)$ for charged particles is found to increase almost linearly with $p_{t}$ at low transverse momentum. At high transverse momentum $v_{2}\left(p_{t}\right)$ deviates from a linear dependence and becomes more flat. Also, heavier particles have smaller $v_{2}\left(p_{t}\right)$ than lighter particles at a given low $p_{t}$, while they seem to have similar values of elliptic flow at higher $p_{t}$. We further find that the increase of the elliptic flow from $\sqrt{s}=130-200 \mathrm{~A} \mathrm{GeV}$ is quite modest in the centrality dependence and is even less in the dependence on transverse momenta below $2 \mathrm{GeV} / c$.

In the present study, we have adopted a simple approach in converting strings to partons and in the hadronization from partons to hadrons. To treat this more consistently, we need to consider the local parton and string densities and determine when strings are converted to partons and partons are 
combined to from hadrons. In a probably more systematic approach, the initial energy may be separated into two parts with the hard part corresponding to minijet gluons and quarks, and the soft part corresponding to color fields. A parton cascade including a color mean field can then be used to describe the subsequent dynamics. Furthermore, both inelastic parton scatterings and particle subdivision $[38,21,39]$ have been neglected in the parton cascade. These may affect the elliptic flow and need to be studied in the future.

\section{ACKNOWLEDGMENTS}

We appreciate useful discussions with U. Heinz, B. A. Li, T. W. Ludlam, M. Murray, J. Y. Ollitrault, Subrata Pal, D. H. Rischke, N. Xu, and B. Zhang. This work was supported by the U.S. National Science Foundation under Grant Nos. PHY-9870038 and PHY-0098805, the Welch Foundation under Grant No. A-1358, and the Texas Advanced Research Program under Grant No. FY99-010366-0081.
[1] J. Barrette et al., E877 Collaboration, Phys. Rev. Lett. 73, 2532 (1994).

[2] H. Appelshauser et al., NA49 Collaboration, Phys. Rev. Lett. 80, 4136 (1998).

[3] J.Y. Ollitrault, Phys. Rev. D 46, 229 (1992).

[4] H. Sorge, Phys. Lett. B 402, 251 (1997); Phys. Rev. Lett. 78, 2309 (1997); 82, 2048 (1999).

[5] P. Danielewicz, R.A. Lacey, P.B. Gossiaux, C. Pinkenburg, P. Chung, J.M. Alexander, and R.L. McGrath, Phys. Rev. Lett. 81, 2438 (1998).

[6] Y. Zheng, C.M. Ko, B.A. Li, and B. Zhang, Phys. Rev. Lett. 83, 2534 (1999).

[7] K.H. Ackermann et al., STAR Collaboration, Phys. Rev. Lett. 86, 402 (2001).

[8] R.A. Lacey, PHENIX Collaboration, Nucl. Phys. A698, 559 (2002).

[9] R.J. Snellings, STAR Collaboration, Nucl. Phys. A698, 193 (2002).

[10] C. Adler et al., STAR Collaboration, Phys. Rev. Lett. 87, 182301 (2001).

[11] C. Roland et al., PHOBOS Collaboration, Nucl. Phys. A698, 54 (2002); I.C. Park et al., PHOBOS Collaboration, ibid. A698, 564 (2002).

[12] H. Heiselberg and R. Mattiello, Phys. Rev. C 60, 044902 (1999).

[13] M. Gyulassy, I. Vitev, and X.N. Wang, Phys. Rev. Lett. 86, 2537 (2001).

[14] X.N. Wang, Phys. Rev. C 63, 054902 (2001).

[15] P. Huovinen, P.F. Kolb, U. Heinz, P.V. Ruuskanen, and S.A. Voloshin, Phys. Lett. B 503, 58 (2001).

[16] P. Huovinen, P.F. Kolb, and U. Heinz, Nucl. Phys. A698, 475 (2002).

[17] E.E. Zabrodin, C. Fuchs, L.V. Bravina, and A. Faessler, Phys. Lett. B 508, 184 (2001).

[18] D. Teaney, J. Lauret, and E.V. Shuryak, Phys. Rev. Lett. 86, 4783 (2001); Nucl. Phys. A698, 479 (2002).

[19] H. Sorge, Phys. Rev. C 52, 3291 (1995).
[20] B. Zhang, M. Gyulassy, and C.M. Ko, Phys. Lett. B 455, 45 (1999).

[21] D. Molnar, and M. Gyulassy, Nucl. Phys. A698, 379 (2002); nucl-th/0104073.

[22] B. Zhang, C.M. Ko, B.A. Li and Z.W. Lin, Phys. Rev. C 61, 067901 (2000).

[23] Z.W. Lin, S. Pal, C.M. Ko, B.A. Li, and B. Zhang, Phys. Rev. C 64, 011902 (2001).

[24] Z.W. Lin, S. Pal, C.M. Ko, B.A. Li, and B. Zhang, Nucl. Phys. A698, 375 (2002); also see http://nt3.phys.columbia.edu/ people/zlin/ZLIN/PUBLICATIONS/lin-qm01.pdf

[25] B.A. Li and C.M. Ko, Phys. Rev. C 52, 2037 (1995).

[26] S.A. Bass et al., Prog. Part. Nucl. Phys. 41, 225 (1998).

[27] X.N. Wang and M. Gyulassy, Phys. Rev. D 44, 3501 (1991); M. Gyulassy and X.N. Wang, Comput. Phys. Commun. 83, 307 (1994).

[28] B. Zhang, C.M. Ko, B.A. Li, Z.W. Lin, and B. Sa, Phys. Rev. C 62, 054905 (2000).

[29] D. Kharzeev and M. Nardi, Phys. Lett. B 507, 121 (2001).

[30] B. Andersson, G. Gustafson, G. Ingelman, and T. Sjostrand, Phys. Rep. 97, 31 (1983); B. Andersson, G. Gustafson, and B. Soderberg, Z. Phys. C 20, 317 (1983).

[31] T. Sjostrand, Comput. Phys. Commun. 82, 74 (1994). Program updates and documentation can be found at http:// www.thep.lu.se/tf2/staff/torbjorn/Pythia.html

[32] B. Zhang, Comput. Phys. Commun. 109, 193 (1998).

[33] We have checked that numerically the violation of total energy in any event is between $-0.4 \%$ and $1.7 \%$ for 5000 minimumbias $\mathrm{Au}+\mathrm{Au}$ events at $130 \mathrm{~A} \mathrm{GeV}$.

[34] M. Gyulassy, Y. Pang, and B. Zhang, Nucl. Phys. A626, 999 (1997).

[35] A. Dumitru, and M. Gyulassy, Phys. Lett. B 494, 215 (2000).

[36] S.A. Voloshin, and A.M. Poskanzer, Phys. Lett. B 474, 27 (2000).

[37] H. Heiselberg and A. Levy, Phys. Rev. C 59, 2716 (1999).

[38] B. Zhang, M. Gyulassy, and Y. Pang, Phys. Rev. C 58, 1175 (1998).

[39] S. Cheng et al., Phys. Rev. C 65, 024901 (2002). 